\title{
Urine Metabolomics in Hemorrhagic Shock: Normalization of Urine in the Face of Changing Intravascular Fluid Volume and Perturbations in Metabolism
}

Elizabeth R. Lusczek ${ }^{1 *}$, Teresa Nelson ${ }^{2}$, Daniel Lexcen ${ }^{1}$, Nancy E. Witowski ${ }^{1}$, Kristine E. Mulier ${ }^{1}$ and Greg Beilman ${ }^{1}$

${ }^{1}$ University of Minnesota, Critical Care and Acute Care Surgery, 516 Delaware St. SE, Minneapolis, MN 55455 USA

${ }^{2}$ Technomics Research, 1815 Medina Rd, Suite 101, Minneapolis, MN 55356 USA

\begin{abstract}
There are many ways to normalize biofluid metabolomics data to account for changes in dilution, all of which have been thoroughly examined in model systems. Here, urine metabolomics data was examined under relevant physiological conditions obtained from a porcine model of hemorrhagic shock and resuscitation. This includes highly variable intravascular fluid volume and urine output coupled with large perturbations in the abundance of endogenous metabolites. Seven different normalization techniques and raw data were evaluated to determine an appropriate normalization technique in this setting, including spectral post-processing methods and physiological measures of concentration. Relationships between normalization constants for each urine sample were examined, as well as relationships between urinary and serum creatinine concentrations. Principal components analysis was used to examine clustering of metabolomics data. The set of normalization constants associated with each sample were reflective of urine concentration, with a trend toward concentration decreases during late resuscitation timepoints. Urinary creatinine normalized to urine output was most reflective of serum creatinine levels. Principal components analysis showed that urine samples clustered according to experimental timepoint for all normalization methods examined. Little separation was seen in raw data. Urine output-normalized data stands out from the six other normalization methods studied because it is reflective of renal clearance and should be used when comparing urine and serum metabolomics data.
\end{abstract}

Keywords: Hemorrhagic Shock; Metabolomics; Urine; Normalization; NMR; Creatinine

Abbreviations: NMR: Nuclear Magnetic Resonance; TSP: 3-(Trimethylsilyl)propionic acid; NOESY: Nuclear Overhauser Effect Spectroscopy; FFT: Fast Fourier Transform; lac: Lactate; cr: Creatinine; CS: Constant Sum; CS-LGU: Constant Sum computed without Lactate, Glucose, and Urea signals; TSI: Total Spectral Intensity; PQ: Probabilistic Quotient; OSM: Osmolality; UO: Urine output; rmANOVA: repeated measures analysis of variance; PCA: principal components analysis; PC1: principal component 1; PC2: principal pomponent 2; PC3: principal component 3; IQR: interquartile range

\section{Introduction}

Hemorrhagic shock, defined by severe loss of an organism's fluid volume, is a leading cause of death in both military and civilian trauma victims $[1,2]$. When bleeding and coagulopathy are controlled, hemorrhagic shock can be reversed by restoring oxygen delivery to the organism using volume replacement intervention and supportive care to control bleeding. Despite these strategies, a number of patients still progress to irreversible hemorrhagic shock and death. The cellular mechanisms leading to complications from shock, such as acute respiratory distress syndrome, systemic inflammatory response syndrome, multiple organ dysfunction syndrome, and multiple organ failure [3] are poorly understood. Even so, ischemia and dysoxia are known to greatly alter the metabolic rate, physiologic mechanisms, and biological mediators involved in the associated pathophysiology [2].

Metabolomics is a new field of study that proposes to outline the entire metabolome, or repertoire of small molecules, in an organism. Using proven analytical chemistry techniques such as Nuclear Magnetic Resonance (NMR) spectroscopy and mass spectrometry, metabolites are identified and quantified from a biological sample of an organism in a given physiological state [4]. The metabolome responds to changes in the physiological state of an organism, and certain metabolites in the metabolome may be indicative of the organism's response to stimuli, health or disease, or response to injury. Metabolomics has been used to investigate metabolic markers of ischemia/reperfusion injury in the kidney, heart, and intestine $[5,6,7,8,9,10]$.

Biofluids such as urine and serum are gaining favor over tissue samples in metabolomic studies because they are easily obtained. However, data from biofluids, especially urine, must be accurately normalized to account for dilution effects. The issue of how to account for dilution changes has been studied extensively $[11,12,13,14,15]$ and relative strengths and weaknesses of different methods have been described. Unfortunately, the most common normalization method, constant sum or integral normalization, may not be robust enough to handle samples with such widely changing individual metabolite concentrations as are known to arise from hemorrhagic shock [11]. Most importantly, the majority of evaluation of normalization techniques has been done with computer simulations of NMR data, or with spectra obtained from known chemical mixtures with known dilutions. There has not been an examination of the behavior of different normalization methods in hemorrhagic shock and resuscitation, a physiological state that encompasses large shifts in intravascular volume, drastic unknown

*Corresponding author: Elizabeth R. Lusczek, MS, University of Minnesota Critical Care and Acute Care Surgery, 516 Delaware St. SE, Minneapolis, MN 55455 USA, Tel:(612) 624-3940; Fax: (612) 625-3675; E-mail: lusc0006@umn.edu

Accepted February 15, 2011; Published February 22, 2011

Citation: Lusczek ER, Nelson T, Lexcen D, Witowski NE, Mulier KE, et al. (2011) Urine Metabolomics in Hemorrhagic Shock: Normalization of Urine in the Face of Changing Intravascular Fluid Volume and Perturbations in Metabolism. J Bioana Biomed 3: 038-048. doi:10.4172/1948-593X.1000041

Copyright: (c) 2011 Lusczek ER, et al. This is an open-access article distributed under the terms of the Creative Commons Attribution License, which permits unrestricted use, distribution, and reproduction in any medium, provided the original author and source are credited. 
Citation: Lusczek ER, Nelson T, Lexcen D, Witowski NE, Mulier KE, et al. (2011) Urine Metabolomics in Hemorrhagic Shock: Normalization of Urine in the Face of Changing Intravascular Fluid Volume and Perturbations in Metabolism. J Bioanal Biomed 3: 038-048. doi:10.4172/1948593X.1000041

changes in urine output and urine concentration, and systematic perturbations in the abundance of endogenous metabolites.

An understanding of changes in the urinary metabolome during hemorrhagic shock and resuscitation has the potential to illuminate the cellular adaptations taking place during this process. However, specific physiological alterations due to hemorrhagic shock complicate the analysis with respect to scaling and normalization. There are substantial changes in fluid volume and urine concentration due to blood loss, followed by dilution of the urine when resuscitation fluids are administered. As a result, urine output fluctuates throughout the course of shock and resuscitation [16]. Additionally, large variations in metabolites associated with shock such as lactate, glucose, and urea means that application of integral normalization techniques are questionable in this setting.

The normalization methods examined here include five spectral post-processing normalization methods and two physiological estimates of urine concentration. The data set was comprised of 83 timed urine samples collected from 13 pigs subjected to hemorrhagic shock and resuscitation. Normalization constants for each sample were plotted against experimental timepoint to assess whether the normalization constants were reflective of expected changes in urine dilution. Urinary creatinine concentrations were compared with serum creatinine concentrations to test each normalization method's effect on the raw creatinine concentrations. Pairwise correlations of the normalization constants were compared with each other to highlight similarities and differences in their relative estimation of concentration. Finally, principal components analysis was used on a subset of the normalized metabolomic data to ensure that reasonable clustering of the data and reasonable proportions of variance were explained. In doing so, this paper examines the behavior of seven different normalization methods in the physiologically relevant setting of hemorrhagic shock described above in order to find an appropriate normalization method.

\section{Experimental}

\section{Animal preparation and hemorrhagic shock protocol}

A well-established model of porcine hemorrhagic shock, which has been previously described [17], was utilized. Male Yorkshire pigs (Manthei Hog Farm, LLC, Elk River, MN) weighing between 15-20 kg were instrumented and splenectomized. A captive bolt device was used to create a blunt percussive injury to the chest. Hemorrhagic shock was then induced by withdrawal of blood from the inferior vena cava until a systolic pressure of the lower 50's was reached (typically 35-50 \% of total blood volume). Shed blood was placed in an acid-citrate-dextrose bag for later use. A liver crush injury was induced using a Holcomb clamp technique [18], with two crush injuries created in the liver parenchyma. Animals received lactated Ringer's fluid given as $20 \mathrm{cc} / \mathrm{kg}$ intravenous (IV) boluses to maintain a systolic blood pressure of greater than 80 $\mathrm{mmHg}$ for one hour of limited resuscitation; then underwent full resuscitation by protocol (see Figure 1). Auto-transfused warmed blood was given at $10 \mathrm{cc} / \mathrm{kg}$ IV boluses for a target hemoglobin of greater than $6 \mathrm{~g} / \mathrm{dL}$, and, a urine output of greater than $1 \mathrm{cc} / \mathrm{kg} / \mathrm{hr}$ was targeted using lactated Ringer's at $20 \mathrm{cc} / \mathrm{kg}$ IV boluses or blood at $10 \mathrm{cc} / \mathrm{kg} /$ $\mathrm{hr}$. Serum creatinine levels were obtained at set timepoints from $1 \mathrm{~mL}$ aliquots of blood via rate reflectance spectrophotometry calibrated to isotope dilution mass spectrometry (Fairview Diagnostic Laboratories, Minneapolis, MN USA). After the resuscitation period, animals were extubated and sent to recovery. At 24-48 hours after resuscitation, animals were re-intubated for endpoint sample harvesting and then euthanized.

\section{Experimental Timeline}

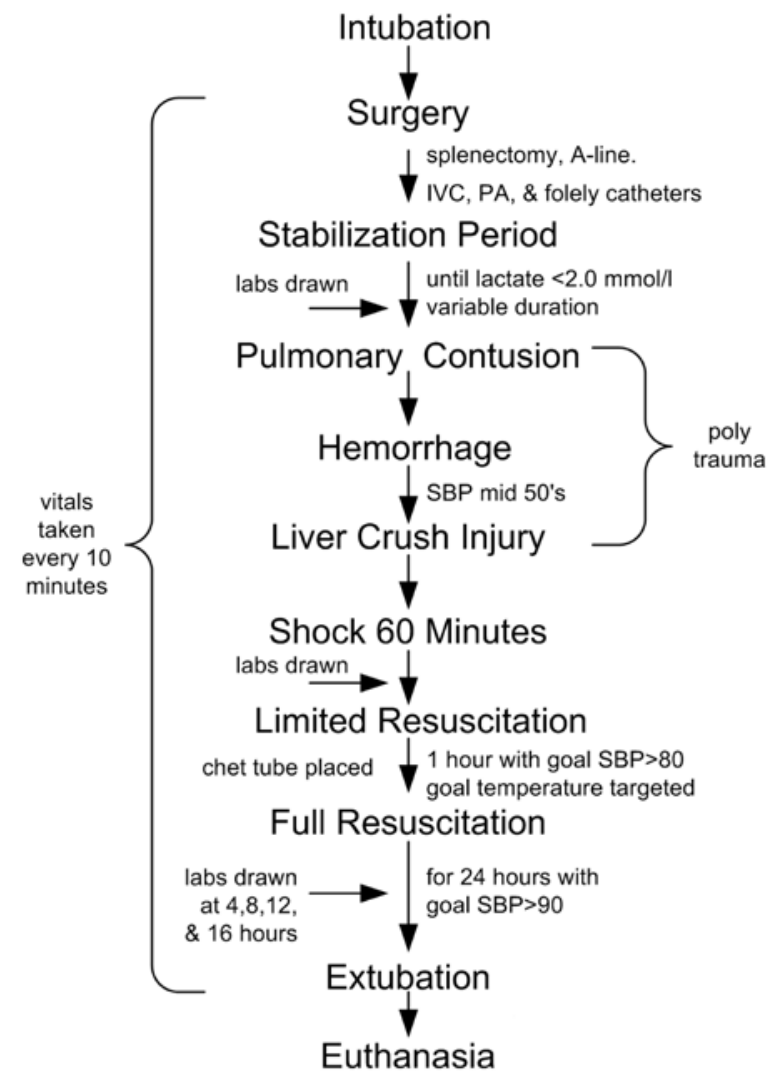

Figure 1: Graphical representation of the resuscitation algorithm employed in both experiments, as discussed in the Materials and Methods.

Urine samples from seven animals were taken from a study performed in 2008, and samples from six animals were taken from a similar study (2009-2010). All experimental protocols and instrumentation were the same for the two experiments; the only difference was the experimental timepoints at which urine samples were obtained (see Table 1). A total of 83 urine samples were used in this study.

\section{Identification of metabolites from NMR}

Urine samples were taken at the time intervals specified in Table 1 from the Foley catheter and were frozen and stored at $-80^{\circ} \mathrm{C}$. Samples were thawed at the time of preparation for NMR analysis. One $\mathrm{mL}$ of thawed urine was mixed with $0.5 \mathrm{~mL}$ of $0.2 \mathrm{M}$ sodium phosphate buffer prepared with $\mathrm{D} 2 \mathrm{O}$ to control $\mathrm{pH}$. The mixture was placed on ice for 10 minutes and then centrifuged at $7000 \mathrm{~g}$ for 10 minutes. $500 \mu \mathrm{L}$ of the supernatant was withdrawn and combined with $50 \mu \mathrm{L}$ of the internal standard 3-(Trimethylsilyl)propionic acid (TSP, Sigma-Aldrich, USA) to a concentration of $1 \mathrm{mM}$ [19]. The internal standard was prepared with $\mathrm{D} 2 \mathrm{O}$ to provide a lock for the NMR signal. The $\mathrm{pH}$ of the final solution was recorded (mean 7.62, standard deviation 0.22 ) and the mixture was transferred to separate $5 \mathrm{~mm}$ NMR tubes (Wilmad, LabGlass, USA).

Proton NMR spectra were taken with a Bruker Avance spectrometer with autosampler and $5 \mathrm{~mm}$ triple resonance $1 \mathrm{H} / 13 \mathrm{C} / 15 \mathrm{~N}$ TXI CryoProbe with Z-gradient, running TopSpin v. 2.16 (Bruker BioSpin, 
Citation: Lusczek ER, Nelson T, Lexcen D, Witowski NE, Mulier KE, et al. (2011) Urine Metabolomics in Hemorrhagic Shock: Normalization of Urine in the Face of Changing Intravascular Fluid Volume and Perturbations in Metabolism. J Bioanal Biomed 3: 038-048. doi:10.4172/1948$593 X .1000041$

\begin{tabular}{|c|c|c|c|c|c|c|c|c|c|c|}
\hline & \multirow{2}{*}{ Baseline } & \multirow{2}{*}{ Shock 45} & \multicolumn{7}{|c|}{ Time in hours after shock } & \multirow[b]{2}{*}{$24 / 48$} \\
\hline & & & 1 & 2 & 4 & 8 & 12 & 16 & 20 & \\
\hline $2008(n=7)$ & $x$ & $x$ & $\mathrm{X}$ & & $\mathrm{X}$ & $\mathrm{X}$ & $\mathrm{X}$ & $x$ & $\mathrm{X}$ & $x$ \\
\hline $2009-2010(n=6)$ & $x$ & $x$ & & $x$ & & $x$ & & & $x$ & $x$ \\
\hline Timepoint & 1 & 2 & 3 & 4 & 5 & 6 & 7 & 8 & 9 & $10 / 11$ \\
\hline Phase & Pre-shock & Shock & \multicolumn{3}{|c|}{ Early Resuscitation } & \multicolumn{4}{|c|}{ Late Resuscitation } & End \\
\hline
\end{tabular}

Table 1: Experimental time points between the two experiments. Baseline/timepoint 1 samples were taken after instrumentation. Shock 45/timepoint 2 samples were taken after 45 minutes of hemorrhagic shock. LR1/timepoint 3 samples were taken after one hour of resuscitation; FR2/timepoint 4 samples were taken after 2 hours of resuscitation, etc. FR20/timepoint 9 samples indicate the end of resuscitation. Animals in the 2008 experiments had experimental endpoints at 24 or 48 hours after the end of resuscitation (PR24/timepoint 10 or PR48/timepoint 11). Animals in the 2009-2010 experiments had experimental endpoints of 48 hours after the end of resuscitation (PR48/timepoint 11)

Fremont, CA USA) at 700.13 MHz (Figure 2). A 1D NOESY (Nuclear Overhauser Effect Spectroscopy) pulse sequence was used. The $90^{\circ}$ pulse width was calibrated for each sample, and was generally $12-13 \mu$ s. The relaxation time was defined by each sample's $90^{\circ}$ pulse width. The relaxation delay was $2 \mathrm{~s}$, the acquisition time was $3 \mathrm{~s}$, the spectral width was $10 \mathrm{kHz}$, the total number of data points collected was 63,000, and the number of transients collected was 128 , for a total experiment time of 11 minutes and 17 seconds. During the relaxation period, the water resonance was presaturated. All spectra were collected at a temperature of $298 \mathrm{~K}$. Line broadening at $0.5 \mathrm{~Hz}$ was applied before FFT; autophasing and auto-baseline correction were applied by TopSpin.

Chenomx software [20] was used to identify and quantify a portion of the metabolites present in each urine sample. Fine manual phasing and baseline corrections and the software's Reference Deconvolution algorithm were applied to each spectrum before targeted profiling of the metabolites was performed. Fifty-seven metabolites were fit in each urine sample in this study, resulting in a profile containing the concentration of each identified metabolite in millimoles per liter $(\mathrm{mM})$. The metabolomic profiles containing the urine concentrations were then normalized in seven different ways described below.

\section{Normalization of urinary metabolites}

Each urine sample has seven normalization constants associated with it, one for each method of normalization, with the exception of urine output as stated below.

Constant Sum (CS): This post-processing method controls for the varying concentration in the urine by dividing the concentration of each metabolite by the sum of the concentration for all the metabolites profiled with Chenomx in a given sample. This method assumes that each metabolite's concentration is increased by the same amount due to the increased concentration of the urine sample. The normalized metabolite abundances are expressed as a fraction of profiled metabolites.

Constant Sum, excluding lactate, glucose, and urea concentrations (CS-LGU): This post-processing method is the same as CS; however, lactate, glucose, and urea concentrations are left out of the calculation of the normalization constants because the concentrations of these metabolites are highly variable during the experiment. The units of the normalized metabolite abundances are the same as for CS normalization.

Total Spectral Intensity (TSI): The assumptions for this postprocessing method are the same as for constant sum normalization. Each spectrum was divided into bins of width $0.04 \mathrm{ppm}$, and standardized to the spectral area under the TSP peak. Bins were summed over the entire spectral area between 0 and $10 \mathrm{ppm}$, excluding the spectral regions containing water and urea to approximate the total area covered by the spectrum. The raw metabolite concentrations are divided by this quantity and the normalized quantity is expressed as a fraction of spectral intensity.

Probabilistic Quotient (PQ1 and PQ2): In these two postprocessing methods [12], a "reference sample" representing the median concentration of the urine is constructed and used to calculate the normalization constants for each sample. Two different methods were used to construct the reference sample. The first method (PQ1) used the metabolite concentrations from the baseline urine samples as the reference sample for the balance of that pig's experimental samples. The second method (PQ2) used each metabolites median concentration across all pigs at baseline as the reference sample, i.e., a hypothetical pig at baseline. The hypothetical pig at baseline was used as the reference sample for all experimental samples. Both PQ1 and PQ2 normalization constants are unitless, so metabolite concentrations normalized to PQ1 and PQ2 also have units of millimoles per liter (mM).

Osmolality (OSM): Osmolality is a direct measure of the concentration of solute particles in the urine. Aliquots of urine were tested for osmolality using micro-osmometry by freezing point depression, with values reported as the number of solute particles in moles dissolved in a kilogram of urine. Metabolite concentrations were normalized by dividing by the osmolality of the appropriate sample. The value returned is a ratio of the abundance of a given metabolite relative to the total number of solute particles. To return a normalized metabolite concentration of nanomoles of metabolite per millimoles of solute, each urine sample's osmolality has been mulitplied by a factor of $1 \times 10^{-6}$.

Urine Output (UO): Urine output quantifies the volume of urine excreted per hour per kilogram of pig mass. Normalization to urine output is performed by multiplying raw metabolite concentrations by urine output, whereas raw metabolite concentration is divided by the normalization constant for all other methods. For this reason, when modeling normalization constants, the inverse of urine output is used. Metabolite abundances normalized to urine output have units of nanomoles of metabolite per hour per $\mathrm{kg}$ of pig mass ( $\mathrm{nmol} / \mathrm{hr} / \mathrm{kg}$ ). Urine output data was not available at experimental endpoint $(24 / 48$ hours after the initiation of shock).

Analysis Method: Seven of the physiological parameters (lactate, arterial $\mathrm{pH}$, base excess, heart rate, mean arterial pressure, cardiac output, and hemoglobin) that are known to change with hemorrhagic shock were compared with repeated measures analysis of variance (rmANOVA) for both experiments to justify pooling the data. The analysis is shown in Appendix 1.

Repeated measures ANOVA were used to estimate the average normalization constant for each timepoint collected. Covariance structures (auto-regressive, unspecified or compound symmetry) were used to account for the correlation expected among the samples collected for each pig when the model indicated a statistically significant fit. Variance components were used otherwise. Tukey's 
Citation: Lusczek ER, Nelson T, Lexcen D, Witowski NE, Mulier KE, et al. (2011) Urine Metabolomics in Hemorrhagic Shock: Normalization of Urine in the Face of Changing Intravascular Fluid Volume and Perturbations in Metabolism. J Bioanal Biomed 3: 038-048. doi:10.4172/1948$593 \times .1000041$

adjustment was used to correct for the multiple pairwise comparisons made to determine the reason for the statistical significance when found. Spearman rank correlation analysis was used to compare the normalization constants in a pairwise manner.

Urinary creatinine levels were compared to serum creatinine levels to test the behavior of the normalization methods. Appearance of creatinine in the urine should follow that of serum levels because it is freely filtered by the kidneys. However, this is complicated by intravascular volume, with higher intravascular volumes typically resulting in higher urine output and thus a lower urinary creatinine concentration. Creatinine levels, which correlate with renal blood flow in healthy kidneys, should accumulate in the blood as a result of decreased renal handling and reduced intravascular volume from hemorrhagic shock. A robust normalization technique should preserve this behavior. Estimated mean creatinine concentrations in the serum and urine for each normalization method plus raw concentrations were calculated by rmANOVA with error bars to indicate the $95 \%$ confidence interval.

Principal components analysis (PCA), a standard metabolomics analysis tool, was used to reduce data dimensionality and identify key metabolites responsible for variation in the physiology studied. The urinary metabolomic data sets were standardized in preparation for PCA. Singular value decomposition was used, and PCA was performed on the correlation matrix derived from the standardized metabolomic data. For each method of normalization, PCA with metabolite reduction was performed up to 4 times to identify influential observations and eliminate them from the dataset. The method used to reduce the number of metabolites included in the PCA was that recommended by Joliffe [21]. Influential observations were clearly identified on the PC1 (principal component 1) by PC2 (principal component 2) score plot; samples that were outliers were clearly separated from the rest of the observations. To confirm a sample's outlier status, the PC loadings and concentrations for the metabolites in a sample were reviewed. In each case, the samples in question contained metabolites that loaded strongly in PC1 or PC2. The metabolite concentrations in these samples were at least 1.5 interquartile ranges (IQR) beyond the IQR, a standard definition of a statistical outlier.

\section{Results}

Normalization constants were analyzed by timepoint with rmANOVA. The results of Tukey's multiple range tests are shown as the estimated mean normalization constant and $95 \%$ confidence interval (Figure 3). The result shows a decrease in the magnitude of the normalization constants after resuscitation $(\mathrm{p} \leq 0.0556)$ in PQ1 and PQ2 (timepoints 7-9), OSM (timepoint 8), and UO (timepoints 7-8). Additionally, baseline UO was significantly higher than at timepoints 4 and 6-9. No significant pairwise comparisons between timepoints were observed in CS, CS-LGU, or TSI.

Creatinine values for serum and urine under all normalization methods are shown in Figure 4. Serum creatinine levels were found to vary significantly between experiments, with a greater rise in creatinine levels between timepoints 3 and 10 for the 2009-10 experiments compared to 2008 (Figure 4A). The difference in average creatinine between experiments, while statistically significant, is not clinically relevant.

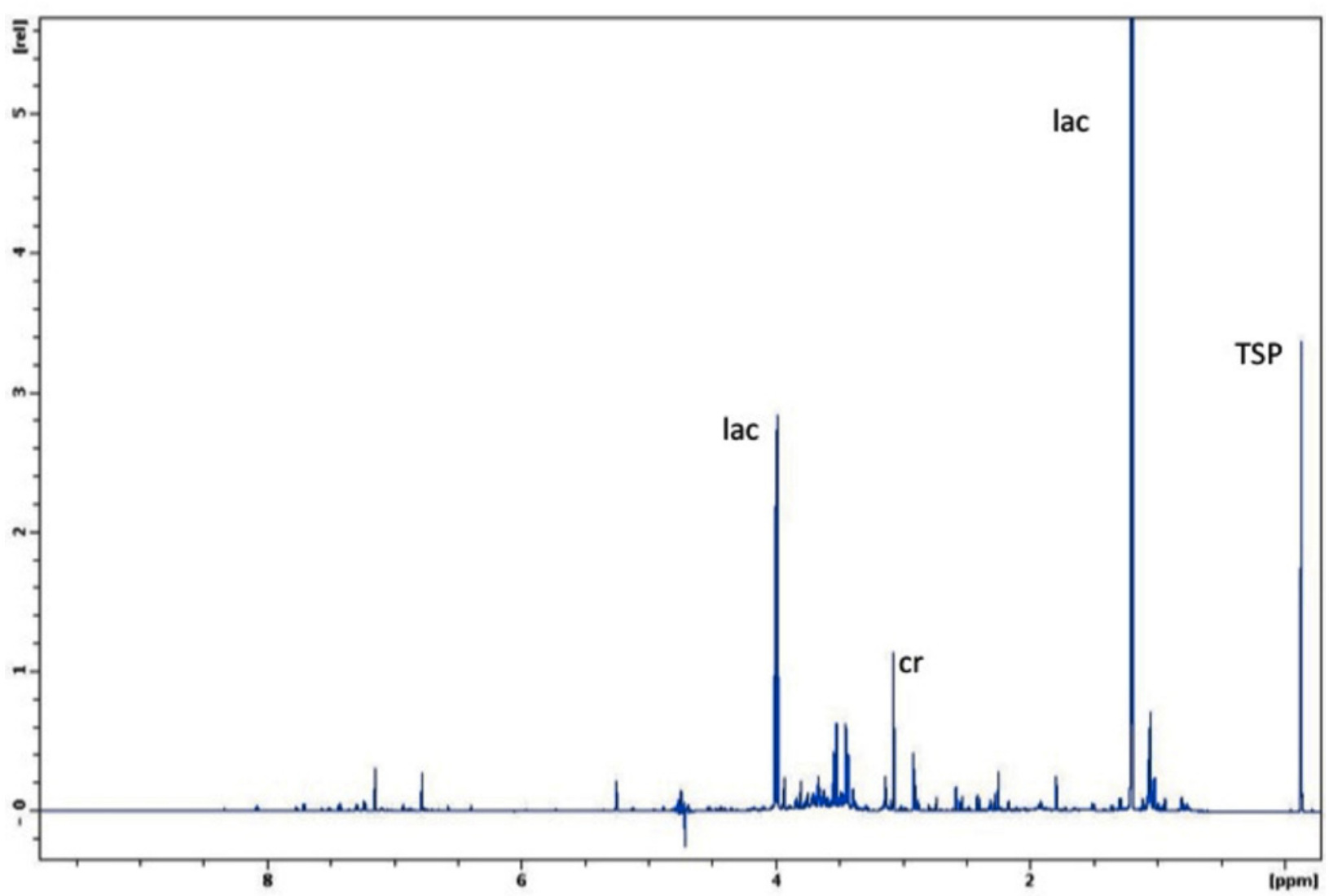

Figure 2: NMR spectrum of a representative urine sample taken on a $700 \mathrm{MHz}$ Bruker Avance Spectrometer with NOESY pulse sequence. 
Citation: Lusczek ER, Nelson T, Lexcen D, Witowski NE, Mulier KE, et al. (2011) Urine Metabolomics in Hemorrhagic Shock: Normalization of Urine in the Face of Changing Intravascular Fluid Volume and Perturbations in Metabolism. J Bioanal Biomed 3: 038-048. doi:10.4172/1948$593 X .1000041$

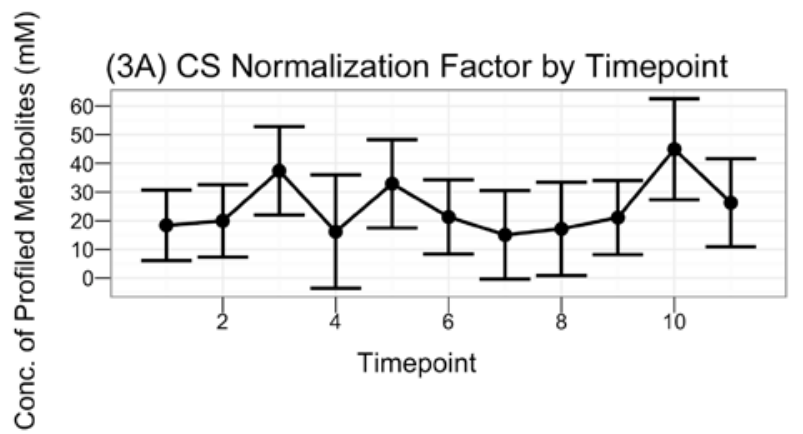

(3C) TSI Normalization Factor by Timepoint

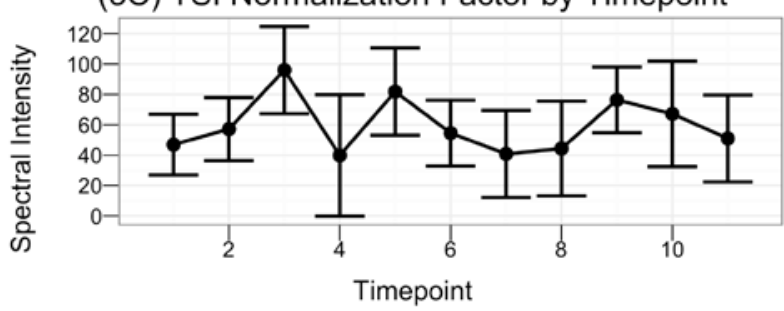

(3E) PQ2 Normalization Factor by Timepoint
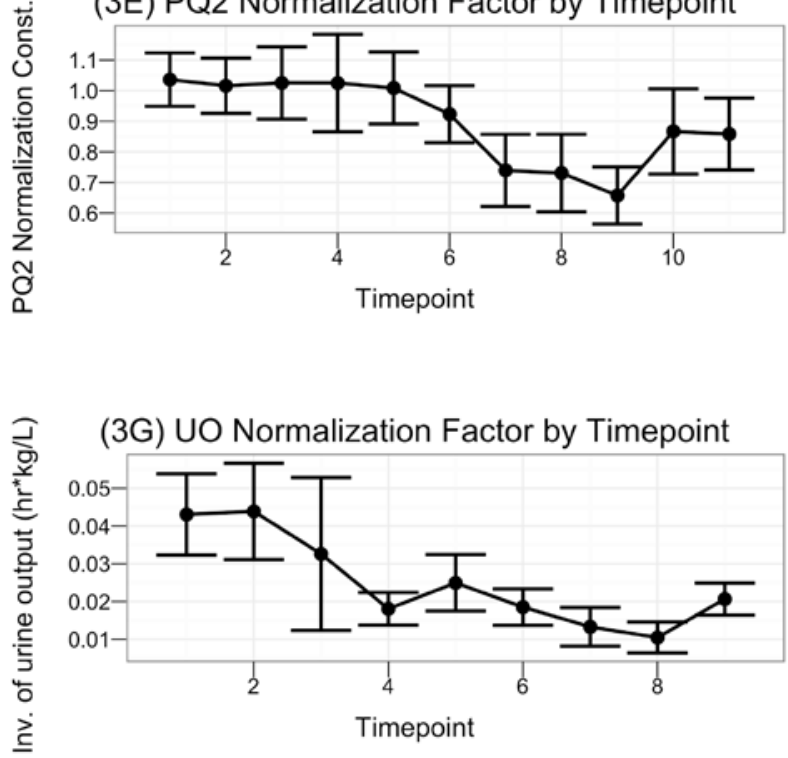

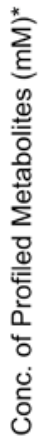

(3B) CS-LGU Normalization Factor by Timepoint

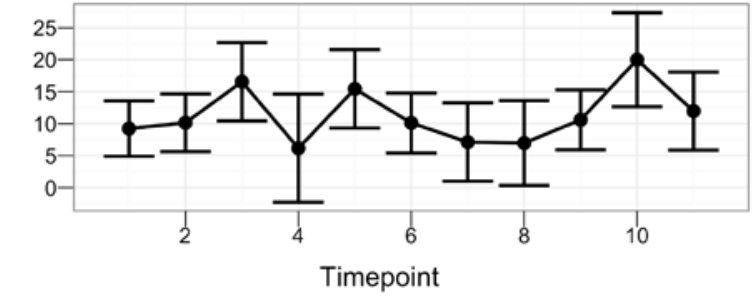

(3D) PQ1 Normalization Factor by Timepoint
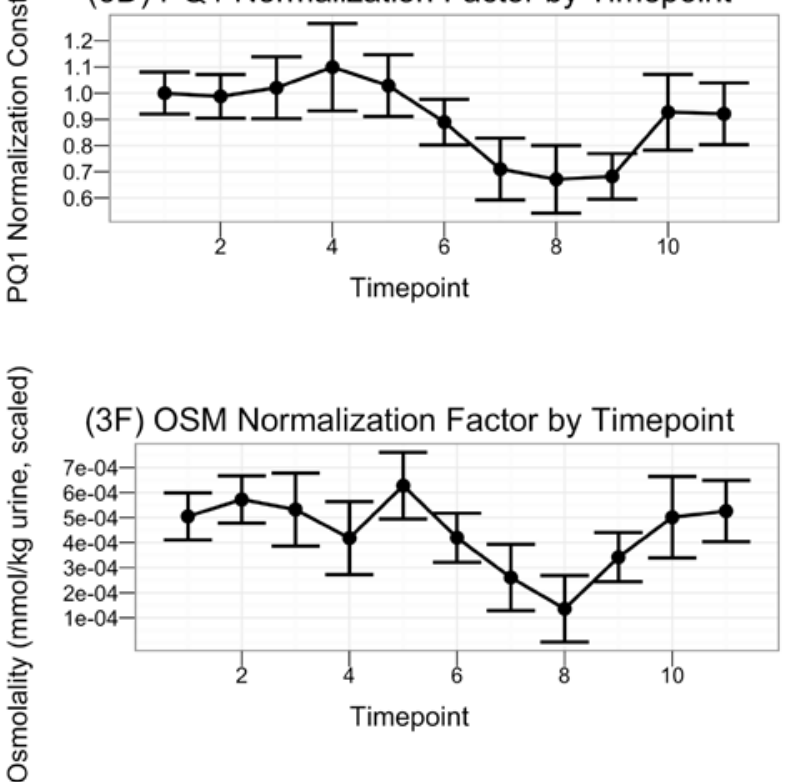

Figure 3: The seven plots of the estimated mean normalization constant with error bars to indicate the $95 \%$ confidence interval for the mean as estimated by rmANOVA. (3A): Constant sum normalization constants (mM) plotted against experimental timepoint. (3B): Constant Sum, excluding lactate, glucose, and urea concentrations (mM, *: exclusive of lactate, glucose, and urea concentrations) normalization constants plotted against experimental timepoint. (3C): Total spectral intensity (arbitrary units, obtained by spectral binning) normalization constants plotted against experimental timepoint. (3D) and (3E): PQ1 and PQ2 normalization constants (unitless) plotted against experimental timepoint. (3F): OSM normalization constants ( $m$ mol of solvent per kg of urine, scaled by a factor of $1 \times 10^{-6}$ ), plotted against experimental timepoint. (3G): Urine output normalization constants ( $\mathrm{hr} \mathrm{kg} / \mathrm{nmol}$ ) plotted against experimental timepoint. The UO normalization constant is the inverse of the animal's urine output.

Urinary creatinine levels did not vary between experiments; however, they did vary with normalization method (Figures $4 \mathrm{~B}-\mathrm{I}$ ). Urinary creatinine normalized to UO follows the same general pattern as serum creatinine though they are not the same $(\mathrm{p}<0.0001)$. Urine normalized to CS, CS-LGU, and TSI suggest the opposite pattern ( $\mathrm{p}<$ 0.0001 ), with a decrease in normalized creatinine abundance between baseline and endpoint. There is little change with time in the abundance of urinary creatinine normalized to PQ1 ( $<<0.0001)$, PQ2 $(\mathrm{p}<0.0001)$, and OSM $(\mathrm{p}<0.0779)$. The statistical significance for PQ1 and PQ2 is due to the reduction in variability and decreased magnitude observed at timepoint 4.

Spearman rank correlation coefficients were calculated for each pair of normalization constants. Plots of the normalization constants against each other, r-values, and p-values are shown in Figure 5. The 
Citation: Lusczek ER, Nelson T, Lexcen D, Witowski NE, Mulier KE, et al. (2011) Urine Metabolomics in Hemorrhagic Shock: Normalization of Urine in the Face of Changing Intravascular Fluid Volume and Perturbations in Metabolism. J Bioanal Biomed 3: 038-048. doi:10.4172/1948593X.1000041

correlations indicate that CS, CS-LGU, and TSI are correlated $(\mathrm{r} \geq 0.79$, $\mathrm{p}<0.0001)$. The PQ methods are correlated with each other $(\mathrm{r}=0.67$, $\mathrm{p}<0.0001)$, but not with CS, CS-LGU, or TSI $(\mathrm{r} \leq|0.18|, \mathrm{p}>0.11)$. UO and OSM are also correlated with each other $(\mathrm{r}=0.65, \mathrm{p}<0.001)$. Thus, CS, CS-LGU, and TSI are related, PQ1 and PQ2 are related, and OSM and UO are related. UO has at least a moderate correlation to all other normalization methods, and is the only method to have a nearly significant $\mathrm{p}$-value $(\mathrm{p}<0.051)$ associated with every other normalization method. OSM is comparable in this regard, though there is no statistical significance with PQ2 ( $\mathrm{p}=0.12)$.

Given the similarities observed in calculation and findings between CS/CS-LGU/TSI, PQ1/PQ2, and OSM/UO, it was decided that principal components analysis would not be completed on urinary metabolomic data normalized to CS, TSI, and PQ1. Only CS-LGU, PQ2, OSM, UO, and the raw data would be analyzed by PCA. The number of samples removed due to influential status via up to four rounds of PCA with metabolite reduction varied from method to method (see Appendix 2). These samples were clear statistical outliers for the metabolites loading heavily on the relevant PC (PC1 or PC2). Experimental notes were reviewed to find a non-statistical justification for removal of the outliers, and none was found.

Table 2 shows the metabolites and number of PCs retained by each method, the eigenvalues, the cumulative eigenvectors/loadings, and the proportion of variation explained for each PC. Thirteen metabolites were not retained by any of the normalization methods or in the raw data. The number of metabolites retained varied from 12-20 and the proportion of variation explained ranged from $35 \%$ to $66 \%$. Of the four normalization methods, UO described the most variation, at $54 \%$ in two principal components.

Two and three-dimensional (2 retained PCs and 3 retained PCs, respectively) scores plots were reviewed for clustering (see Figure 6). All four normalization methods appeared to cluster the late resuscitation timepoints separately from the other timepoints, with slight differences between the normalization methods. Little meaningful clustering of the raw urine data was observed.

\section{Discussion}

Urine is often used in metabolomics studies because it is readily available. However, changes in dilution confound the quantification of metabolite concentrations in urine samples. This problem is exacerbated in hemorrhagic shock, where urine concentrations are known to vary widely. Additionally, large variations in endogenous metabolites are often involved in the systemic response to hemorrhagic shock. These variations can further confound some of the normalization methods that are commonly used.

Seven methods for normalizing urine metabolomics data were examined in a porcine model of hemorrhagic shock and resuscitation. Evaluation criteria were that the normalization constants reflect expected increases and decreases in urine concentration and that normalized urinary creatinine levels reflect serum creatinine levels. Normalization methods were also compared for similarities in urine concentration estimation using Spearman correlation. Methods that were different were analyzed by PCA and compared with PCA of the raw data to check for meaningful separation of the data.

Analysis of the normalization constants by timepoint with rmANOVA indicates that the constants associated with each urine sample are reflective of the sample's concentration. While there was no indication of a significant increase in urine concentration as a consequence of hemorrhage, there was a trend toward decrease in urine concentration after resuscitation for all normalization methods and a significant decrease in urine concentration in all methods except CS, CS-LGU, and TSI. This is at least partially due to the administration of resuscitation fluids after timepoint 2 , and the subsequent increase of intravascular fluid volume. The decrease in urine concentration is generally observed during later time points in the hemorrhagic shock model (Timeponts 6-9).

Serum creatinine levels varied between the two experiments pooled in this study. Serum creatinine levels are known to be variable even in healthy individuals [22]. Both groups present a consistent pattern with lower serum creatinine levels at baseline and at timepoint 11 with elevated levels after shock. Normal serum creatinine values for weaner pigs ( $<40 \mathrm{lbs}$ ) range from $0.75-1.95 \mathrm{mg} / \mathrm{dL}$ and values for feeder pigs (40-80 lbs) range from $0.79-1.87 \mathrm{mg} / \mathrm{dL}$ [23]. Ultimately, the differences observed here between experiments are small $(0.2-0.3 \mathrm{mg} / \mathrm{dL})$ relative to the width of the normal range. Such differences are considered clinically insignificant.

Urinary creatinine normalized to UO most closely tracks serum creatinine. There is a slight depression of urinary creatinine levels relative to serum creatinine during shock, which is likely reflective of reduced blood flow to the kidney after shock and reduced filtration capabilities of the nephron [24]. When compared to UO-normalized creatinine, urinary creatinine under the other normalizations displays little change over the course of the experiment (PQ1, PQ2, OSM), or displays the opposite behavior (CS, CS-LGU, TSI; see Figure 4).

The discrepancy between UO and the other normalization methods arises from the resulting time-dependence of normalization by urine output, which expresses urinary creatinine levels as nanomoles per hour

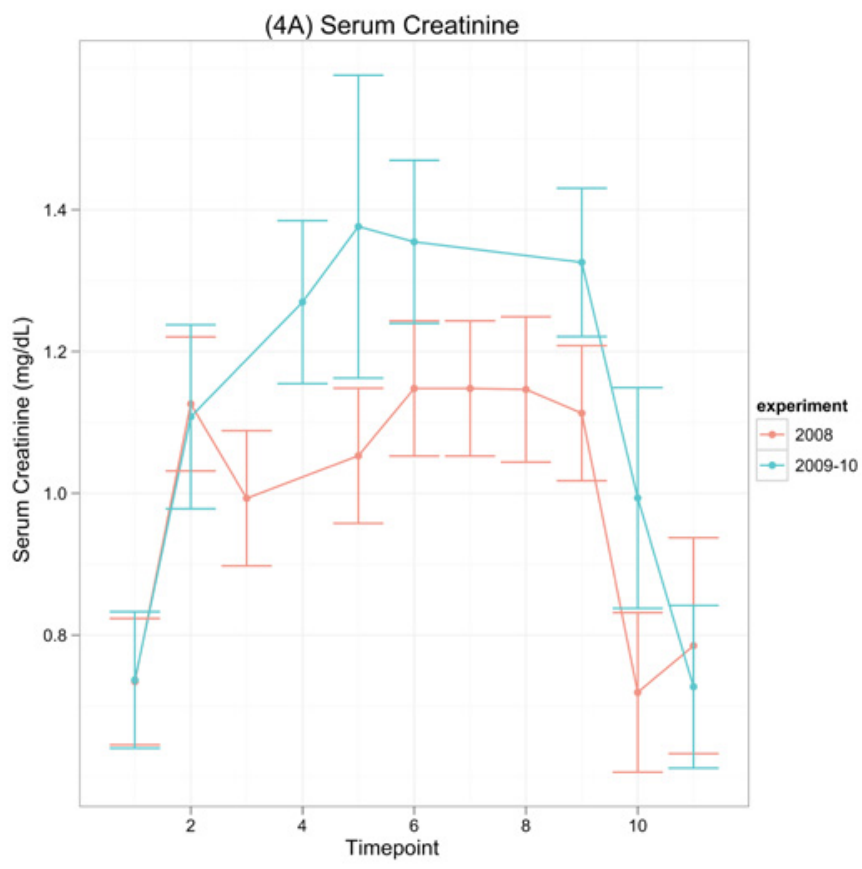

Figure 4: The nine stacked plots illustrate the estimated mean creatinine concentration in the serum (A) and urine (B-I) for each normalization method plus raw. Error bars indicate the $95 \%$ confidence interval for the mean as estimated by rmANOVA. *: Profiled metabolites are exclusive of lactate, glucose, and urea. **: Units are $\mathrm{mM}$ but scaled by the appropriate $\mathrm{PQ}$ normalization constant. 
Citation: Lusczek ER, Nelson T, Lexcen D, Witowski NE, Mulier KE, et al. (2011) Urine Metabolomics in Hemorrhagic Shock: Normalization of Urine in the Face of Changing Intravascular Fluid Volume and Perturbations in Metabolism. J Bioanal Biomed 3: 038-048. doi:10.4172/1948593X.1000041

per kilogram of pig mass. This is reflective of the clearance of creatinine from the blood, a decidedly time-dependent phenomenon. No other normalization method returns a time-dependent value of metabolite abundance in the urine; therefore, no other method can yield a result that is concordant with renal clearance. The clear relationship between serum creatinine levels and creatinine clearance as indicated by the urinary creatinine values normalized to UO distinguishes it from all other normalization methods. This observation should not lead to outright rejection of other methods, however. The urinary creatinine levels returned by the remaining normalization methods are reflective of creatinine levels at a given timepoint. Serum creatinine is observed to rise in the blood as a result of reduced filtering capabilities of the nephron from ischemic kidney damage. Because of this, the total amount of creatinine excreted in the urine will be diminished. This is observed in CS, CS-LGU, and TSI-normalized urine (Figure 4 C-E).

Each normalization method estimates the urine concentration differently, and the correlation analysis of the normalization constants
(Figure 5) highlights these differences. The two physiological measures of urine dilution, OSM and UO, are well-correlated with each other These two methods are at best moderately correlated with the postprocessing methods, save OSM with CS-LGU and TSI. Still, UO and OSM have the strongest correlations with the other methods, indicating an underlying physiological mechanism to the estimation of concentration.

It is observed that CS, CS-LGU, and TSI are highly correlated with each other because these normalization constants are all reflective of NMR spectral intensity. PQ1 and PQ2 are well-correlated with each other because both methods rely on median estimates of concentration. However, the normalization constants for PQ1 and PQ2 are only moderately correlated with UO and OSM, and are not correlated with CS, CS-LGU, and TSI. The PQ methods and the integral methods (CS, CS-LGU, TSI) should be well correlated unless there is some systematic variation in the metabolite abundances across the samples [12,15]. This is the case in a hemorrhagic shock state.

\section{Spearman Correlations and p-values of Normalization Constants}

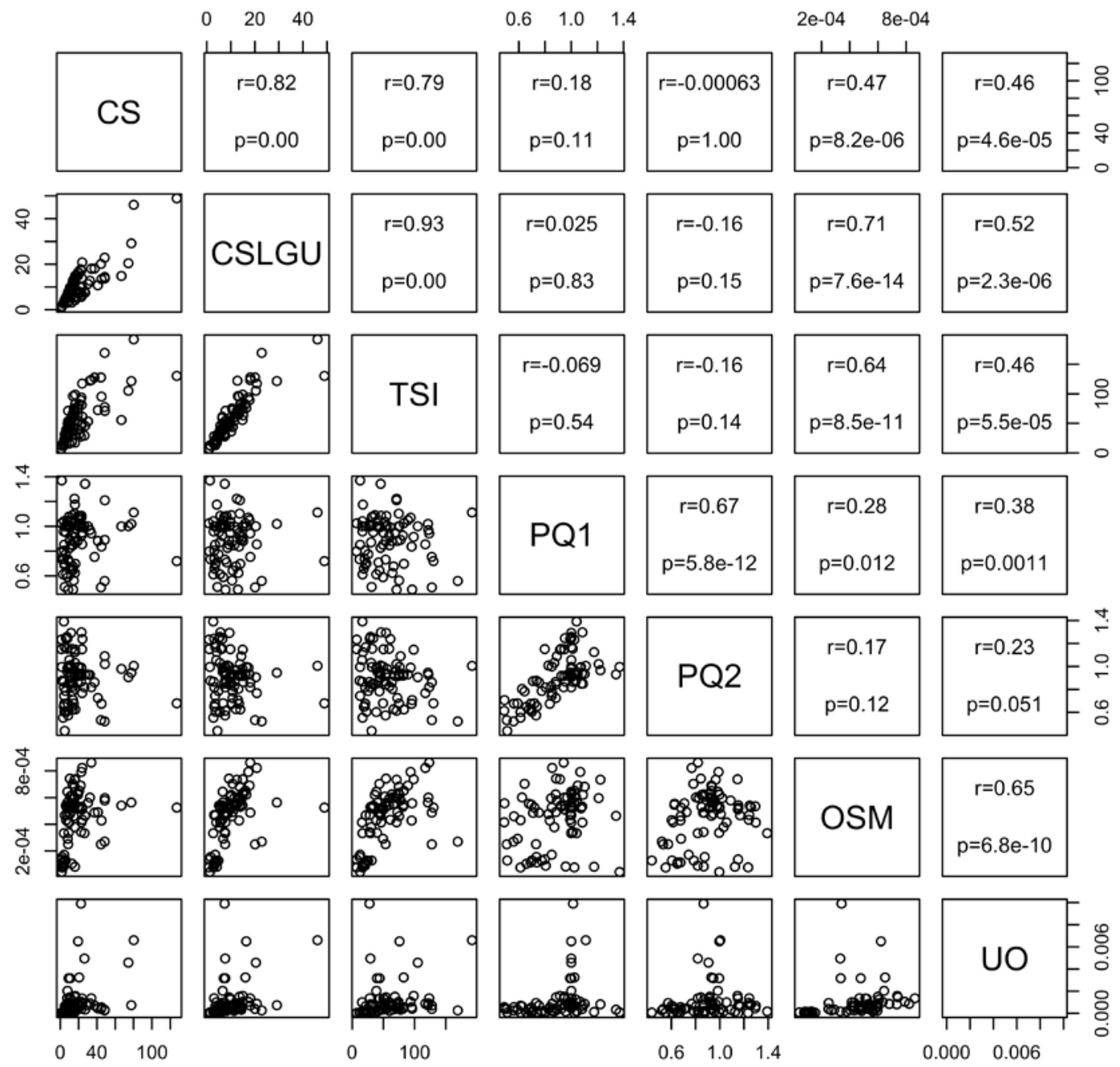

Figure 5: Scatter plots of each of the normalization constants against each other (below diagonal) with Spearman correlation r-values and p-values (above diagonal) Scales on the plots vary with the constants as described in Figure 2: The plotting convention is as follows: the graph in the second row of the figure plots the CS normalization constants on the x-axis and the CS-LGU normalization constants on the y-axis. 
Citation: Lusczek ER, Nelson T, Lexcen D, Witowski NE, Mulier KE, et al. (2011) Urine Metabolomics in Hemorrhagic Shock: Normalization of Urine in the Face of Changing Intravascular Fluid Volume and Perturbations in Metabolism. J Bioanal Biomed 3: 038-048. doi:10.4172/1948593X.1000041

The three major contributors to endogenous metabolite variation in this data set are urinary lactate, glucose, and urea. Contributions from each of these metabolites were removed from the CS-LGU normalization constant. The urea signal was also removed from the TSI normalization constant because of complications from the water suppression, as is standard practice with spectral binning [25].

There are both physiological and experimental sources of metabolite variation in this model that must be considered. Lactate is a well-studied marker of traumatic injury [26, 27]. Lactate levels are seen to vary from $0.004 \mathrm{mM}$ to $15.33 \mathrm{mM}$ in the raw data, with the highest concentrations occurring at timepoints 3 and 4 (1-2 hours after shock). It is also known that hemorrhagic shock produces alterations in energy metabolism [28] that make additional contributions to systematic metabolite variation. In this case, glucose is observed to fluctuate over a range of $0.014-18.93 \mathrm{mM}$ in the raw data. The kidney's handling of lactate may also be involved in glucose variation [29]. Finally, urea, which can build up in the blood under conditions of decreased perfusion, ranges from $0.22-76.8 \mathrm{mM}$ in the raw data. This, too, has a physiological basis, as blood urea levels are known to increase in trauma patients. Blood urea levels are used as a nonspecific indicator of renal function if the kidneys are unable to clear it $[29,30]$. As a consequence, low fractional excretion of urea is often observed in patients with impaired kidney function [31,32]. Additionally, urea protons are in exchange with water. From an experimental standpoint, suppression of the water signal in

\begin{tabular}{|c|c|c|c|c|c|c|c|c|c|c|c|c|}
\hline \multirow{3}{*}{$\begin{array}{l}\text { Metabolites Retained by at } \\
\text { Least One Metho }{ }^{d} 1\end{array}$} & \multicolumn{12}{|c|}{$\begin{array}{l}\text { Loadings (Multiplied by } 100 \text {, rounded to nearest integer) } \\
\text { Black indicates metabolite not retained by method }\end{array}$} \\
\hline & \multicolumn{3}{|c|}{ Raw } & \multicolumn{3}{|c|}{ CSLGU } & \multicolumn{2}{|c|}{ PQ2 } & \multicolumn{2}{|c|}{ OSM } & \multicolumn{2}{|c|}{ INV UO } \\
\hline & PC1 & PC2 & PC3 & PC1 & PC2 & PC3 & PC1 & PC2 & PC1 & PC2 & PC1 & PC2 \\
\hline 1Methylnicotinamide & & & & & & & 7 & 45 & -12 & 52 & & \\
\hline 2Methylglutarate & 35 & -0 & -4 & & & & & & & & & \\
\hline 2Oxoglutarate & & & & 13 & -4 & 49 & & & 7 & 5 & 24 & 12 \\
\hline 4Hydroxybenzoate & 29 & -4 & -23 & & & & & & & & & \\
\hline Acetate & & & & & & & 19 & 29 & & & & \\
\hline Alanine & 33 & 9 & 21 & -2 & 35 & 28 & & & & & & \\
\hline Allantoin & 26 & -17 & -36 & & & & & & & & & \\
\hline Ascorbate & 33 & 1 & -24 & & & & 30 & 13 & 42 & 13 & & \\
\hline Betaine & & & & -18 & 38 & -11 & & & & & 39 & -25 \\
\hline Citrate & & & & 26 & -4 & 44 & 7 & 26 & & & & \\
\hline Creatine & 8 & 45 & 14 & -28 & 7 & 7 & 29 & -43 & & & 33 & 14 \\
\hline Creatinine & & & & 36 & -25 & -25 & & & & & & \\
\hline Formate & & & & & & & 28 & -34 & 6 & -40 & & \\
\hline Glucitol & & & & -2 & 36 & -1 & & & & & & \\
\hline Glucose & & & & 11 & 36 & -32 & & & & & -4 & -0 \\
\hline Glutamate & & & & 23 & 11 & 23 & & & & & & \\
\hline Glutamine & & & & & & & & & 42 & 22 & & \\
\hline Glycerol & & & & 3 & 13 & 28 & & & 33 & -19 & & \\
\hline Glycine & & & & -22 & 14 & 6 & 36 & -22 & & & & \\
\hline Hippurate & & & & & & & 20 & 4 & & & 26 & -42 \\
\hline Homogentisate & & & & -15 & 27 & 3 & & & & & & \\
\hline Hypoxanthine & & & & & & & & & & & & \\
\hline Inosine & & & & & & & 35 & 6 & & & 31 & 37 \\
\hline Isoleucine & 36 & 5 & 7 & & & & 37 & 18 & 36 & -0 & & \\
\hline Lactate & 11 & -28 & 57 & & & & & & & & -1 & 30 \\
\hline Mannitol & & & & & & & & & & & 9 & 41 \\
\hline Mannose & & & & 14 & 32 & -29 & & & 14 & 17 & & \\
\hline Methylguanidine & & & & 39 & 16 & -14 & & & & & & \\
\hline NIsovaleroylglycine & & & & & & & 32 & 28 & & & & \\
\hline NNDimethylglycine & & & & & & & & & 22 & 38 & & \\
\hline Niacinamide & 14 & -38 & 2 & 28 & 20 & 5 & & & & & & \\
\hline Oxypurinol & & & & & & & & & 12 & -1 & & \\
\hline Phenylacetylglycine & & & & 25 & -0 & -9 & & & 22 & 4 & 39 & 3 \\
\hline Pyruvate & 21 & -33 & 40 & & & & & & 1 & 23 & & \\
\hline Quinolinate & 30 & 10 & -6 & & & & & & & & & \\
\hline Taurine & & & & & & & & & 34 & -23 & 32 & -45 \\
\hline Trigonelline & & & & 37 & -3 & -4 & & & & & 24 & 39 \\
\hline Trimethylamine & 14 & 44 & 14 & & & & 29 & -33 & & & 36 & -12 \\
\hline Trimethylamine N-Oxide & 15 & 44 & 9 & & & & & & 12 & -42 & & \\
\hline Tyrosine & & & & 11 & 25 & 22 & & & & & & \\
\hline Urea & 22 & -11 & -35 & & & & & & 28 & 1 & & \\
\hline Valine & 33 & -4 & 21 & & & & 29 & 23 & & & & \\
\hline Xanthine & & & & 3 & -19 & -0 & & & & & 28 & 35 \\
\hline Xanthosine & & & & 25 & 13 & 1 & & & 19 & 2 & & \\
\hline Eigenvalue & 5.44 & 2.60 & 1.89 & 3.86 & 2.49 & 1.78 & 4.57 & 2.37 & 3.36 & 2.24 & 5.02 & 1.43 \\
\hline Cumulative Proportion Explained & $36 \%$ & $54 \%$ & $66 \%$ & $19 \%$ & $32 \%$ & $41 \%$ & $35 \%$ & $53 \%$ & $21 \%$ & $35 \%$ & $42 \%$ & $54 \%$ \\
\hline
\end{tabular}

Table 2: Results of PCA analysis including metabolites retained for raw and normalized urine samples, eigenvectors/loadings, eigenvalues, and proportion of variation explained. The metabolites not retained by any method include 1-6-Anhydro- $\beta$-D-glucose, Adenosine, Choline, cis-Aconitate, Dimethylamine, Fumarate, Glutathione, Glycoproline, Lysine, Proline, Succinate, Tryptophan, and Tyramine. 
Citation: Lusczek ER, Nelson T, Lexcen D, Witowski NE, Mulier KE, et al. (2011) Urine Metabolomics in Hemorrhagic Shock: Normalization of Urine in the Face of Changing Intravascular Fluid Volume and Perturbations in Metabolism. J Bioanal Biomed 3: 038-048. doi:10.4172/1948593X.1000041

proton NMR will render the urea signal unreliable. All three of these metabolites were removed from the CS-LGU normalization constant in an attempt to control for variation. Given the lack of correlation with this method and PQ1/PQ2, it was not sufficient. Other metabolites could very well be contributing to the lack of agreement between the CS methods and the PQ methods. One likely candidate is creatine, which is present in very small amounts in timepoints $1-4$, and increases steadily through the experimental endpoint, covering a range of $0.006 \mathrm{mM}$ to $2.9 \mathrm{mM}$ in the raw data. Creatinuria is documented as an indication of traumatic injury [33].

When PCA was applied to the metabolomic data under different normalizations as described, it was found that different methods retained different metabolites. Several metabolites were rejected by all normalization methods (see Table 2). This phenomenon has not been discussed in metabolomics normalization literature.

As discussed, PCA for all of the normalized metabolomic data elevated to this part of the analysis shows some separation of the urine samples by timepoint (Figure 6). Early timepoints, corresponding to baseline (black circles), shock (red triangles), and early resuscitation (green crosses) are separated from late timepoints (blue x's), corresponding to post-resuscitation. The separation of late resuscitation timepoints (6-9) is reflective the decrease in concentration under rmANOVA analysis of the normalization constants. No separation of the data is seen in principal components analysis of the raw data. The similar clustering of the normalized data into pre-resuscitation and post-resuscitation states suggests that 1) some normalization is required to obtain useful clustering of urine metabolomics data, 2) metabolomics may be useful in differentiating between states of preresuscitation and post-resuscitation, and 3) changes occurring in the physiological state of the animals between hemorrhage and postresuscitation can be differentiated by urine alone.

Of the post-processing methods, none stands out as better or worse than another. The CS methods (CS, CS-LGU, TSI) are fairly well-
(6A) PCA of Raw Data

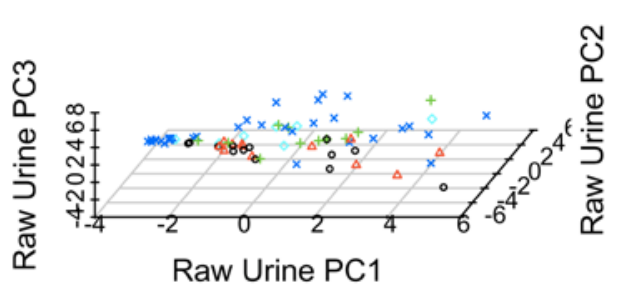

(6C) PCA of PQ2 Normalized Data

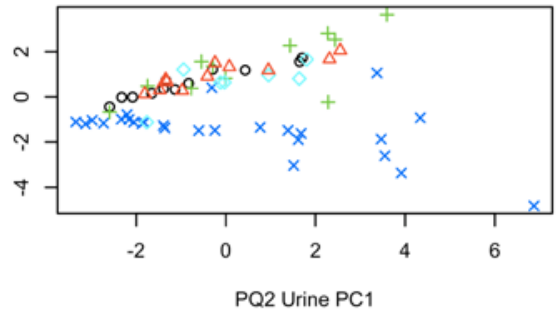

(6E) PCA of UO Normalized Data

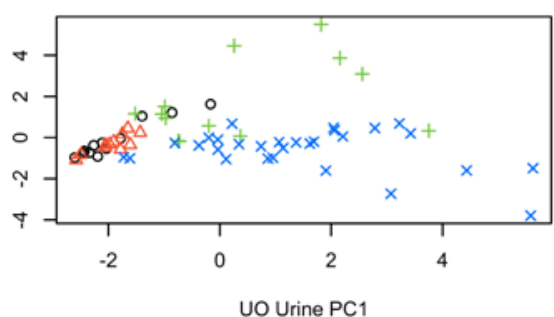

(6B) PCA of CS-LGU Normalized Data

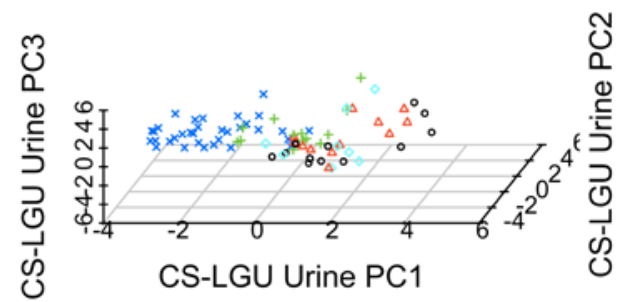

(6D) PCA of OSM Normalized Data

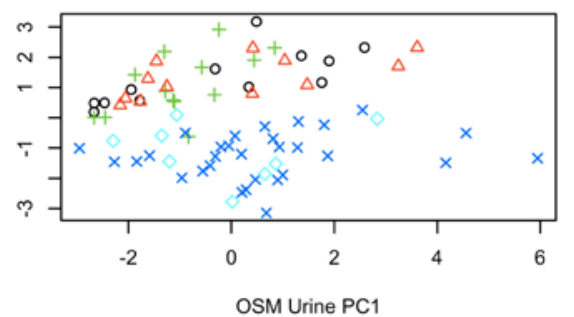

Legend

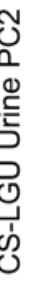

.


Citation: Lusczek ER, Nelson T, Lexcen D, Witowski NE, Mulier KE, et al. (2011) Urine Metabolomics in Hemorrhagic Shock: Normalization of Urine in the Face of Changing Intravascular Fluid Volume and Perturbations in Metabolism. J Bioanal Biomed 3: 038-048. doi:10.4172/1948$593 \times .1000041$

correlated to physiological measures of dilution but it is known that they are not robust to the large shifts in metabolite abundances that are seen in this setting. Additionally, these three methods did not show statistical significance in dilution of the urine at late timepoints (Figure 3). On the other hand, the PQ methods are more robust to drastic systematic changes in metabolism by design. They show a statistically significant decrease in urine concentration at late resuscitation timepoints. However, correlations of normalization constants in Figure 4 indicate that they are different from every other normalization method save each other.

This study demonstrates that UO stands out as being different from the other normalization methods under the examination of creatinine concentrations with time. Creatinine normalized to urine output is reflective of renal clearance; other normalizations of creatinine are not. The inherent time-dependence of renal clearance has been demonstrated. The concentration of solute into urine by the kidneys has been modeled by time-dependent partial differential equations $[34,35]$.

It has been argued that urine output is the only way to obtain concentrations suitable for absolute comparisons of urine metabolite abundance [11]. Therefore, when relationships between urine metabolomic profiles and serum metabolomic profiles are studied, it is suggested that urine must be normalized to UO if meaningful comparisons are to be made. UO also had at least moderate correlations with all other normalization methods, and it is the only normalization method to have a nearly significant $p$-value associated with all other normalization methods. The principal components analysis provides some small additional support for urine output, as it describes the next highest percent of variation in the data set after the raw data.

Not all experimental situations allow for collection of urine output data. In these cases, OSM is preferable, as it is reflective of the physiological metabolite-concentrating mechanisms of the kidney. If osmolality data is not available, one of the post-processing methods would suffice, though care must be taken to apply these methods judiciously. The literature discusses the known issue with Constant Sum and TSI methods-namely, that large perturbations in single metabolite variations can artificially alter normalized metabolite concentrations $[11,12,15]$. If this is not the case, the computationally light CS/TSI methods could be used. However, if there is large systematic variation in metabolite abundances, one of the PQ methods should be used.

It is recognized that the relatively small sample size used $(n=13$ across both experiments) is not ideal. However, the entire sample set is composed of 83 samples. Additionally, urine output data was not available for experimental endpoint (timepoints 10/11). The strength of $\mathrm{UO}$ as a normalization method was apparent despite the lack of endpoint data because of its time dependence.

\section{Concluding Remarks}

A major challenge in metabolomics is the normalization of urine to account for dilution. Hemorrhagic shock exacerbates the problem by inducing severe changes in intravascular volume and urine output, accompanied by large fluctuations in metabolite abundances as a response to injury. To accurately analyze urine metabolomic data, changes in concentration must be accounted for. Seven normalization methods were examined under these conditions to see which would be most appropriate to use in analysis of urine metabolomics data with a porcine model of hemorrhagic shock and resuscitation. While no statistically significant change in concentration is observed after hemorrhage, all methods show a trend toward dilution of the urine after resuscitation fluids are administered. PCA of the normalized metabolite concentrations shows that the normalized data clusters according to experimental timepoint, and consequently by dilution.

Based on this analysis of normalization constants for each sample, serum and urine values of creatinine, and principal components analysis of the metabolite concentrations, it is concluded that urine output normalization is the most appropriate method to use, especially when comparing urine and serum metabolomics data. If urine is being analyzed independently for biomarkers, a different normalization method could be used.

\section{Acknowledgements}

The authors wish to thank the Office of Naval Research for their continued funding of this research (grants N00014-09-1-0323 and N000-05-1-0344), the Minnesota Supercomputing Institute, the University of Minnesota's Nuclea Magnetic Resonance Facility (funding provided by NSF grant BIR-961477, the University of Minnesota Medical School, and the Minnesota Medical Foundation), and the University of Minnesota.

\section{References}

1. Bellamy RF (1984) The causes of death in conventional land warfare: implications for combat casualty care research. Mil Med 149: 55-62.

2. Heckbert SR, Vedder NB, Hoffman W, Winn RK, Hudson LD, et al. (1998) Outcome after hemorrhagic shock in trauma patients. J Trauma 45: 545-549.

3. Jarrar D, Chaudry IH, Wang P (1999) Organ dysfunction following hemorrhage and sepsis: mechanisms and therapeutic approaches (Review). Int J Mol Med. 4: $575-583$.

4. Nicholson JK, Lindon JC, Holmes E (1999) 'Metabonomics': understanding the metabolic responses of living systems to pathophysiological stimul via multivariate statistical analysis of biological NMR spectroscopic data Xenobiotica 29: 1181-1189.

5. Wishart DS (2006) Metabolomics in monitoring kidney transplants. Curr Opin Nephrol Hypertens 15: 637-642.

6. Serkova N, Fuller TF, Klawitter J, Freise CE, Niemann CU (2005) H-NMRbased metabolic signatures of mild and severe ischemia/reperfusion injury in rat kidney transplants. Kidney Int 67: 1142-1151.

7. Turer AT, Stevens RD, Bain JR, Muehlbauer MJ, van der Westhuizen J, et al. (2009) Metabolomic profiling reveals distinct patterns of myocardial substrate use in humans with coronary artery disease or left ventricular dysfunction during surgical ischemia/reperfusion. Circulation 119: 1736-1746.

8. Lv Y, Liu X, Yan S, Liang X, Yang Y, et al. (2010) Metabolomic study of myocardial ischemia and intervention effects of Compound Danshen Tablets in rats using ultra-performance liquid chromatography/quadrupole time-of-flight mass spectrometry. J Pharm Biomed Anal 52: 129-135.

9. Feala JD, Coquin L, Paternostro G, McCulloch AD (2008) Integrating metabolomics and phenomics with systems models of cardiac hypoxia. Prog Biophys Mol Biol 96: 209-225

10. Kinross J, Warren O, Basson S, Holmes E, Silk D, et al. (2009) Intestina ischemia/reperfusion injury: defining the role of the gut microbiome. Biomark Med 3: 175-192.

11. Craig A, Cloarec O, Holmes E, Nicholson JK, Lindon JC (2006) Scaling and normalization effects in NMR spectroscopic metabonomic data sets. Anal Chem 78: 2262-2267.

12. Dieterle F, Ross A, Schlotterbeck G, Senn H (2006) Probabilistic quotien normalization as robust method to account for dilution of complex biological mixtures. Application in $1 \mathrm{H}$ NMR metabonomics. Anal Chem 78: 4281-4290.

13. Warrack BM, Hnatyshyn S, Ott KH, Reily MD, Sanders M, et al. (2009) Normalization strategies for metabonomic analysis of urine samples. J Chromatogr B Analyt Technol Biomed Life Sci 877: 547-552.

14. Webb-Robertson BJ, Lowry DF, Jarman KH, Harbo SJ, Meng QR, et al. (2005) A study of spectral integration and normalization in NMR-based metabonomic analyses. J Pharm Biomed Anal 39: 830-836.

15. Torgrip RJO, Åberg KM, Alm E, Schuppe-Koistinen I, Lindberg J (2008) A note on normalization of biofluid 1D $1 \mathrm{H}-\mathrm{NMR}$ data. Metabolomics 4: 114-121.

16. Gunnar WP, Merlotti GJ, Barrett J, Jonasson O (1986) Resuscitation from 
Citation: Lusczek ER, Nelson T, Lexcen D, Witowski NE, Mulier KE, et al. (2011) Urine Metabolomics in Hemorrhagic Shock: Normalization of Urine in the Face of Changing Intravascular Fluid Volume and Perturbations in Metabolism. J Bioanal Biomed 3: 038-048. doi:10.4172/1948$593 \times .1000041$

hemorrhagic shock. Alterations of the intracranial pressure after normal saline, $3 \%$ saline and dextran-40. Ann Surg 204: 686-692.

17. Mulier KE, Beilman GJ, Conroy MJ, Taylor JH, Skarda DE, et al. (2005) Ringer's ethyl pyruvate in hemorrhagic shock and resuscitation does not improve early hemodynamics or tissue energetics. Shock 23: 248-252.

18. Holcomb JB, Pusateri AE, Harris RA, Charles NC, Gomez RR, et al. (1999) Effect of dry fibrin sealant dressings versus gauze packing on blood loss in grade $\mathrm{V}$ liver injuries in resuscitated swine. J Trauma 46: 49-57.

19. Mortishire-Smith RJ, Skiles GL, Lawrence JW, Spence S, Nicholls AW, et al. (2004) Use of metabonomics to identify impaired fatty acid metabolism as the mechanism of a drug-induced toxicity. Chem Res Toxicol 17: 165-173.

20. Weljie AM, Newton J, Mercier P, Carlson E, Slupsky CM (2006) Targeted profiling: quantitative analysis of $1 \mathrm{H}$ NMR metabolomics data. Anal Chem 78 : 4430-4442.

21. Jolliffe IT (1972) Discarding variables in a principal component analysis. I: Artificial data. Applied Statistics 160-173.

22. Levey AS, Bosch JP, Lewis JB, Greene T, Rogers N, et al. (1999) A more accurate method to estimate glomerular filtration rate from serum creatinine: a new prediction equation. Ann Intern Med 130: 461-470.

23. Friendship RM, Lumsden JH, McMillan I, Wilson MR (1984) Hematology and biochemistry reference values for Ontario swine. Can J Comp Med 48: 390393.

24. Perrone RD, Madias NE, Levey AS (1992) Serum creatinine as an index of renal function: new insights into old concepts. Clin Chem 38: 1933-1953.

25. Slupsky CM, Rankin KN, Wagner J, Fu H, Chang D, et al. (2007) Investigations of the effects of gender, diurnal variation, and age in human urinary metabolomic profiles. Anal Chem 79: 6995-7004.
26. Blow O, Magliore L, Claridge JA, Butler K, Young JS (1999) The golden hour and the silver day: detection and correction of occult hypoperfusion within 24 hours improves outcome from major trauma. J Trauma 47: 964-969.

27. Husain FA, Martin MJ, Mullenix PS, Steele SR, Elliott DC (2003) Serum lactate and base deficit as predictors of mortality and morbidity. Am J Surg 185: 485491.

28. Taylor JH, Beilman GJ, Conroy MJ, Mulier KE, Myers D, et al. (2004) Tissue energetics as measured by nuclear magnetic resonance spectroscopy during hemorrhagic shock. Shock 21: 58-64.

29. Bellomo R (2002) Bench-to-bedside review: lactate and the kidney. Crit Care 6: 322-326.

30. Long CL, Birkhahn RH, Geiger JW, Blakemore WS (1981) Contribution of skeletal muscle protein in elevated rates of whole body protein catabolism in trauma patients. Am J Clin Nutr 34: 1087-1093.

31. Carvounis CP, Nisar S, Guro-Razuman S (2002) Significance of the fractional excretion of urea in the differential diagnosis of acute renal failure. Kidney Int 62: 2223-2229.

32. Vivino G, Antonelli M, Moro ML, Cottini F, Conti G, et al. (1998) Risk factors for acute renal failure in trauma patients. Intensive Care Med 24: 808-814.

33. Threlfall CJ, Maxwell AR, Stoner HB (1984) Post-traumatic creatinuria. J Trauma 24: 516-523.

34. Layton HE, Pitman EB (1994) A dynamic numerical method for models of renal tubules. Bull Math Biol 56: 547-565.

35. Stephenson JL, Mejia R, Tewarson RP (1976) Model of solute and water movement in the kidney. Proc Natl Acad Sci USA 73: 252-256. 